\title{
Exact Solutions of Bose-Einstein Condensate in Linear Magnetic Field and Time-Dependent Laser Field
}

\author{
W.-H. HUANG ${ }^{a, b, *}$, J.-W. MAO ${ }^{a}$ AND W.-G. QIU ${ }^{a}$ \\ ${ }^{a}$ School of Science, Huzhou University, Huzhou 313000, China \\ ${ }^{b}$ Shanghai Institute of Mathematics and Mechanics, Shanghai University, Shanghai 200072, China
}

(Received April 5, 2010)

\begin{abstract}
A generalized $\frac{G^{\prime}}{G}$-expansion method is extended to construct exact solutions for the Gross-Pitaevskii equation with weak bias magnetic and time-dependent laser fields. Many types of exact solutions including hyperbolic function solution, trigonometric function solution and rational exact solution with parameters are obtained. In addition, soliton solutions are found.
\end{abstract}

PACS: 02.30.Jr, 03.65.Ge, 05.45.Yv, 42.65.Tg

\section{Introduction}

Nonlinear evolution equations (NLEEs) are frequently used to model a wide variety of phenomena in physics, chemistry, biology and even in social science field. Seeking and constructing exact solutions especially soliton solutions of NLEEs is one of the most important and essential task in nonlinear science. With the help of exact solutions, when they exist, the nonlinear phenomena can be better understood. In the past decades, various sophisticated methods have been created such as the inverse scattering method [1], the Bäcklund transformations [2], the Darboux transformations [3] and the Hirota bilinear method [4]. Especially, in recent years, with the rapid development of computerized symbolic computation, many direct and effective algebraic methods are proposed such as the homogeneous balance method [5], tanh function method [6], the Jacobi elliptic function expansion method [7], the F-expansion techniques [8], exp-function method [9] and so on. Recently, Wang et al. [10] introduced a new direct method called $\frac{G^{\prime}}{G}$-expansion method for a reliable treatment of the NLEEs. Then this method is generalized and widely used to seek travelling wave solutions, non-travelling wave solutions and coefficient function solutions for many nonlinear physical models [11-14], and even extended to solve NLEE with variable coefficients [15].

The Gross-Pitaevskii equation appears as a relevant model in various areas of physics: nonlinear optics, fluid mechanics, Bose-Einstein condensation (BEC). The realization of BEC of weakly interacting atomic gases stim-

* corresponding author; e-mail: whhuang2002cn@yahoo.com.cn ulates the exploration of nonlinear properties of matter waves [16-19]. Recently the theoretical and experimental studies have focused on the dynamics and stability of dark solitons of two-component BEC. The nonlinear excitations of BEC in magnetic trap can be described by the Gross-Pitaevskii equation [20]

$$
\begin{gathered}
\mathrm{i} \hbar \psi_{\tau}=-\frac{\hbar^{2}}{2 m} \psi_{y y}+\frac{4 \pi \hbar^{2}}{m} a|\psi|^{2} \psi \\
+y\left[n_{1}+n_{2} \sin (\nu t)\right] \psi,
\end{gathered}
$$

where $\tau$ is the time, $y$ is the coordinate, $m$ is the atomic mass, $a$ is the scattering length between atoms, $n_{1}$ and $n_{2}$ are bias magnetic and time-dependent laser fields parameters, respectively. By the following dimensionless transformation:

$$
\begin{aligned}
& u(x, t)=\psi(y, \tau), \tau=\frac{m \varepsilon t}{4 \pi \hbar a}, y=\sqrt{\frac{\varepsilon}{4 \pi a}} x, \\
& b=\sqrt{\frac{\varepsilon}{\pi a}} \frac{m \varepsilon n_{1}}{8 \pi a \hbar^{2}}, \omega=\frac{\nu m \varepsilon}{4 \pi \hbar a}, c=\sqrt{\frac{\varepsilon}{\pi a}} \frac{m \varepsilon n_{2}}{8 \pi a \hbar^{2}},
\end{aligned}
$$

we can rewrite Eq. (1) as

$$
\mathrm{i} u_{t}=-\frac{1}{2} u_{x x}+\varepsilon|u|^{2} u+x[b+c \sin (\omega t)] u,
$$

where $x$ is measured in units of $\varsigma=1 \mu \mathrm{m}$ (a characteristic length unit in this type of experiment), $t$ is in unit of $m \varsigma^{2} / \hbar, u$ is in units of the square root of $n_{0} a^{3}$ (the maximum density in the initial distribution of the condensate), and the interaction constant is defined as $\varepsilon=4 \pi n_{0} a \varsigma^{2}$, with $a$ being the interatomic scattering length. It is very difficult to solve the variable coefficients nonlinear equation. The bright soliton solutions of Eq. (3) have been obtained when $C=0$ [21]. Recently, Liu et al. [20] have derived the exact bright and 
dark soliton solutions of Eq. (3) by applying the inverse scattering method and selecting the appropriate transformation. And, by the tanh method, Li et al. [22] also obtained some new dark soliton solutions. The goal of the present work is to conduct further investigations of the Gross-Pitaevskii equation by the generalized $\frac{G^{\prime}}{G}$ -expansion method. Many new types of exact analytical solutions and soliton solutions are obtained.

\section{The generalized $\left(\frac{G^{\prime}}{G}\right)$-expand method}

First, we give a brief description for the generalized $\left(\frac{G^{\prime}}{G}\right)$-expand method. For a given NLEE with independent variables $X=(x, y, z, \ldots, t)$ and dependent variable $u$ :

$$
\begin{gathered}
F\left(u, u_{t}, u_{x}, u_{y}, u_{z}, \ldots, u_{x t}, u_{y t}, u_{z t},\right. \\
\left.\ldots, u_{t t}, u_{x x}, u_{y y}, u_{z z}, \ldots\right)=0,
\end{gathered}
$$

we suppose that its solution can be expressed by a polynomial in $\left(\frac{G^{\prime}}{G}\right)$ as follows:

$$
u=\sum_{i=1}^{m} \alpha_{i}(X)\left(\frac{G^{\prime}}{G}\right)^{i}+\alpha_{0}(X), \quad \alpha_{m}(X) \neq 0,
$$

where $\alpha_{0}(X), \alpha_{i}(X)(i=1,2, ., m)$ and $\xi=\xi(X)$ are all functions of $X$ to be determined later, $G=G(\xi)$ satisfies a second order linear ordinary differential equation

$$
G^{\prime \prime}+\lambda G^{\prime}+\mu G=0,
$$

where $G^{\prime \prime}=\frac{\partial^{2} G(\xi)}{\partial \xi^{2}}, G^{\prime}=\frac{\partial G(\xi)}{\partial \xi}$, and $\lambda, \mu$ are real constants.

To determine $u$ explicitly, we take the following four steps:

Step 1. Determine the integer $m$ by balancing the highest order nonlinear terms and the highest order partial derivative of $u$ in Eq. (4).

Step 2. Substitute (5) along with Eq. (6) into Eq. (4) and collect all terms with the same order of $\left(\frac{G^{\prime}}{G}\right)$ together, the left hand side of Eq. (4) is converted into a polynomial in $\left(\frac{G^{\prime}}{G}\right)$. Then set each coefficient of this polynomial to zero to derive a set of over-determined partial differential equations for $\alpha_{0}(X), \alpha_{i}(X)$ and $\xi$.

Step 3. Solve the system of over-determined partial differential equations obtained in Step 2 for $\alpha_{0}(X), \alpha_{i}(X)$ and $\xi$ by use of Mathematica or Maple.

Step 4. Use the results obtained in above steps to derive a series of fundamental solutions of Eq. (6) depending on $\left(\frac{G^{\prime}}{G}\right)$, since the solutions of Eq. (6) have been well known for us, then we can obtain exact solutions of Eq. (4).

\section{Exact solutions for the generalized 2D NLSE}

In order to obtain some exact solutions of Eq. (3), we define the complex $u$ field as:

$$
u(x, t)=A(x, t) \mathrm{e}^{\mathrm{i} B(x, t)},
$$

where $A(x, t)$ and $B(x, t)$ are real functions. Substituting $u(x, t)$ into Eq. (3), we find the following coupled equations for the phase $B(x, t)$ and the amplitude $A(x, t)$ :

$$
\begin{gathered}
A_{t}+A_{x} B_{x}+\frac{1}{2} A B_{x x}=0, \\
-A B_{t}+\frac{1}{2}\left(A_{x x}-A B_{x}^{2}\right)-\varepsilon A^{3} \\
-x[b+c \sin (\omega t)] A=0 .
\end{gathered}
$$

According to the balance principle and the generalized $\frac{G^{\prime}}{G}$-expansion method, the solution of Eqs. (8) and (9) can be expressed in the following form:

$$
\begin{aligned}
& A(x, t)=f(t)+h(t) \frac{G(\xi)^{\prime}}{G(\xi)}, \quad \xi=p(t) x+q(t), \\
& B(x, t)=\Gamma(t) x+\Omega(t),
\end{aligned}
$$

where $f(t), h(t), p(t), q(t), \Gamma(t), \Omega(t)$ are functions of $t$ to be determined. Substituting Eqs. (10) and (11) into Eqs. (8) and (9) and collecting all the terms with the same order of $\frac{G^{\prime}}{G}$ together, the left sides of Eqs. (8) and (9) are converted into two polynomials of $x^{i_{1}}\left(\frac{G^{\prime}}{G}\right)^{j_{1}}$ $\left(i_{1}=0,1 ; j_{1}=0,1,2\right)$ and $x^{i_{2}}\left(\frac{G^{\prime}}{G}\right)^{j_{2}}\left(i_{2}=0,1,2\right.$; $\left.j_{2}=0,1,2,3\right)$, respectively. Setting each coefficient of the two polynomials to zero, after simplifying one can finally derive a system of algebraic or first-order ordinary differential equations for $f(t), h(t), p(t), q(t), \Gamma(t)$ and $\Omega(t)$ as follows:

$$
\begin{aligned}
& -h p_{t} k=0, \\
& -h\left(q_{t}+\Gamma p\right)=0, \\
& f_{t}-h q_{t} \mu-\Gamma h p \mu=0, \\
& h_{t}-h q_{t} \lambda-\Gamma h p \lambda=0, \\
& -f \Omega_{t}-\frac{\Gamma^{2} f}{2}-\varepsilon f^{3}+\frac{h p^{2} \lambda \mu}{2}=0, \\
& -l\left[\Gamma_{t}+c \sin (\omega t)+b\right]=0, \\
& -h\left(\Omega_{t}-p^{2} \mu+3 \varepsilon f^{2}+\frac{\Gamma^{2}}{2}-\frac{p^{2} \lambda^{2}}{2}\right)=0, \\
& \frac{3 h p^{2} \lambda}{2}-3 \varepsilon f h^{2}=0, \\
& h p^{2}-\varepsilon h^{3}=0,
\end{aligned}
$$

where $k=1, \lambda, \mu$ and $l=f, h$.

Solving Eqs. (12)-(20) self-consistently, we have a set of conditions on the coefficients and parameters as follows:

$$
\begin{aligned}
& p=C_{1}, \quad h= \pm \frac{C_{1}}{\sqrt{\varepsilon}}, \quad f= \pm \frac{C_{1} \lambda}{2 \sqrt{\varepsilon}}, \\
& \Gamma=\frac{c \cos (\omega t)}{\omega}-b t+C_{2}
\end{aligned}
$$




$$
\begin{aligned}
q & =C_{1}\left(-\frac{c \sin (\omega t)}{\omega^{2}}+\frac{b t^{2}}{2}-C_{2} t+C_{3}\right) \\
\Omega & =-\frac{b^{2}}{6} t^{3}+\frac{1}{2} b C_{2} t^{2}-\frac{1}{4} t\left(2 C_{2}^{2}+C_{1}^{2} \lambda^{2}\right. \\
& \left.+\frac{c^{2}-4 c b \sin (\omega t)}{\omega^{2}}\right)-\frac{c C_{2} \sin (\omega t)}{\omega^{2}}+\frac{c b \cos (\omega t)}{\omega^{3}} \\
& -\frac{c^{2} \cos (2 \omega t)}{8 \omega^{3}}+C_{4},
\end{aligned}
$$

where $C_{1}, C_{2}, C_{3}$, and $C_{4}$ are arbitrary constants. Substituting Eqs. (10) and (11) with Eqs. (21)-(24) into Eq. (7), we have the fundamental solution of Eq. (3):

$$
u= \pm \frac{C_{1} \lambda}{2 \sqrt{\varepsilon}}\left[1+\frac{2}{\lambda}\left(\frac{G(\xi)^{\prime}}{G(\xi)}\right)\right] \exp (\mathrm{i}(\Gamma x+\Omega)),
$$

where

$$
\begin{aligned}
\xi & =p x+q \\
& =C_{1} x+C_{1}\left(-\frac{c \sin (\omega t)}{\omega^{2}}+\frac{b t^{2}}{2}-C_{2} t+C_{3}\right), \\
\Gamma & =\frac{c \cos (\omega t)}{\omega}-b t+C_{2}, \\
\Omega & =-\frac{b^{2}}{6} t^{3}+\frac{1}{2} b C_{2} t^{2}-\frac{1}{4} t\left(2 C_{2}^{2}+C_{1}^{2} \lambda^{2}\right. \\
& \left.+\frac{c^{2}-4 c b \sin (\omega t)}{\omega^{2}}\right)-\frac{c C_{2} \sin (\omega t)}{\omega^{2}}+\frac{c b \cos (\omega t)}{\omega^{3}} \\
& -\frac{c^{2} \cos (2 \omega t)}{8 \omega^{3}}+C_{4}
\end{aligned}
$$

and $G(\xi)$ is the solution of Eq. (6).

Substituting the general solutions of Eq. (6) into Eq. (25), we have three types of exact solutions of Eq. (3) as follows:

$$
\begin{aligned}
u_{1} & = \pm \frac{C_{1} \lambda}{2 \sqrt{\varepsilon}} \\
\times & \left(1+\frac{2}{\lambda} \frac{K_{1} \sinh \left(\frac{\sqrt{\lambda^{2}-4 \mu}}{2} \xi\right)+K_{2} \cosh \left(\frac{\sqrt{\lambda^{2}-4 \mu}}{2} \xi\right)}{K_{1} \cosh \left(\frac{\sqrt{\lambda^{2}-4 \mu}}{2} \xi\right)+K_{2} \sinh \left(\frac{\sqrt{\lambda^{2}-4 \mu}}{2} \xi\right)}\right) \\
& \times \exp (\mathrm{i}(\Gamma x+\Omega)), \quad \lambda^{2}-4 \mu>0, \\
u_{2} & = \pm \frac{C_{1} \lambda}{2 \sqrt{\varepsilon}} \\
\times & \left.+\frac{2}{\lambda} \frac{-K_{1} \sin \left(\frac{\sqrt{4 \mu-\lambda^{2}}}{2} \xi\right)+K_{2} \cos \left(\frac{\sqrt{4 \mu-\lambda^{2}}}{2} \xi\right)}{K_{1} \cos \left(\frac{\sqrt{4 \mu-\lambda^{2}}}{2} \xi\right)+K_{2} \sin \left(\frac{\sqrt{4 \mu-\lambda^{2}}}{2} \xi\right)}\right) \\
\times & \exp (\mathrm{i}(\Gamma x+\Omega)), \quad \lambda^{2}-4 \mu<0,
\end{aligned}
$$

$$
\begin{gathered}
u_{3}= \pm \frac{C_{1} \lambda}{2 \sqrt{\varepsilon}}\left(1+\frac{2}{\lambda} \frac{K_{2}}{K_{1}+K_{2} \xi}\right) \exp (\mathrm{i}(\Gamma x+\Omega)), \\
\lambda^{2}-4 \mu=0,
\end{gathered}
$$

where $K_{1}, K_{2}$ are arbitrary constants, $\varepsilon>0(a>0)$ and $\xi, \Gamma, \Omega$ determined by Eq. (26).

In particular, for the hyperbolic function solution $u_{1}$ expressed by Eq. (27), if setting $K_{2}=0$, we can obtain the following dark soliton solution:

$$
\begin{aligned}
u_{4} & = \pm \frac{C_{1} \lambda}{2 \sqrt{\varepsilon}}\left[1+\frac{2}{\lambda} \tanh \left(\frac{\sqrt{\lambda^{2}-4 \mu}}{2} \xi\right)\right] \\
& \times \exp (\mathrm{i}(\Gamma x+\Omega)), \quad \lambda^{2}-4 \mu>0 .
\end{aligned}
$$

Figure 1 shows the dark soliton solution $U=\left|u_{4}\right|$ in detail with the conditions $C_{1}=C_{2}=C_{3}=b=c=\omega=\varepsilon=1$, $\lambda=3, \mu=1$. When $\lambda= \pm 2, \mu=0$, Eq. (30) is just the soliton solution reported in Ref. [22].

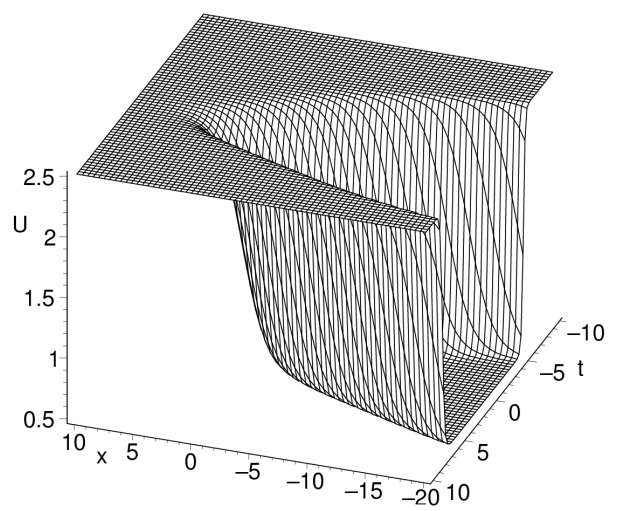

Fig. 1. Dark soliton solution $U=\left|u_{4}\right|$ with $C_{1}=C_{2}=$ $C_{3}=b=c=\omega=\varepsilon=1, \lambda=3, \mu=1$.

\section{Summary and discussion}

To summarize, applying the generalized $\frac{G^{\prime}}{G}$-expansion method, three types of new exact solutions including hyperbolic function solution, trigonometric function solution and rational solution with parameters for the GrossPitaevskii equation with weak bias magnetic and time-dependent laser fields are obtained. Selecting the parameters and the arbitrary functions in the solutions freely, more abundant exact solutions including soliton solutions can be derived. A new dark soliton solution is presented. The more about the physical significance and its stability for the dark soliton solution in BEC for the GrossPitaevskii equation will be investigated somewhere else. The $\frac{G^{\prime}}{G}$-expansion method is generalized and extended to solve variable coefficients nonlinear physical models and shown effective and powerful. More exploring about the solutions for the Gross-Pitaevskii equation and seeking exact solutions for other NLEEs by the generalized $\frac{G^{\prime}}{G}$ -expansion method is further worth studying. 


\section{Acknowledgments}

This work is supported by the Foundation of Zhejiang Education Committee No. Y200908781 and in part by the Natural Science Foundation of China under Grant No. 11072139

\section{References}

[1] M.J. Ablowitz, H. Segur, Soliton and the Inverse Scattering Transformation, SIAM, Philadelphia 1981.

[2] M.R. Miura, Bäcklund Transformation, Springer Verlag, Berlin 1978.

[3] P.G. Esteevez, J. Math. Phys. 40, 1406 (1999).

[4] H.W. Tam, W.X. Ma, X.B. Hu, D.L. Wang, J. Phys. Soc. Jpn. 69, 45 (2002).

[5] M.L. Wang, Phys. Lett. A 216, 67 (1996).

[6] E.J. Perkes, B.R. Duffy, Comp. Phys. Commun. 98, 288 (1996).

[7] S.K. Liu, Z.T. Fu, S.D. Liu, Q. Zhao, Phys. Lett. A 289, 69 (2001).

[8] Y.B. Zhou, M.L. Wang, Y.M. Wang, Phys. Lett. A 308, 31 (2003).

[9] J.H. He, X.H. Wu, Chaos Solitons Fractals 30, 700 (2006).

[10] M.L. Wang, X.Z. Li, J.L. Zhang, Phys. Lett. A 372, 417 (2007).
[11] M. Wang, X. Li, J. Zhang, Appl. Math. Comput. 206 , 321 (2008).

[12] B.Q. Li, Y.L. Ma, Acta Physica Sinica 58, 4373 (2009) (in Chinese).

[13] J. Zhang, X. Wei, Y. Lu, Phys. Lett. A 372, 3653 (2008).

[14] S. Zhang, W. Wang, J.L. Tong, Appl. Math. Comput. 209, 399 (2009).

[15] S. Zhang, J.L. Tong, W. Wang, Phys. Lett. A 372, 225 (2008).

[16] M.H. Anderson, J.R. Ensher, M.R. Matthews, Science 269, 198 (1995).

[17] L. Deng, E.W. Hagley, J. Wen, M. Trippenbach, Y. Band, P.S. Julienne, J.E. Simsarian, K. Helmerson, S.L. Rolston, W.D. Phillips, Nature 398, 218 (1999).

[18] S. Burger, K. Bongs, S. Dettmer, W. Ertmer, K. Sengstock, Phys. Rev. Lett. 83, 5198 (1999).

[19] T.H. Busch, J.R. Anglin, Phys. Rev. Lett. 84, 2298 (2000).

[20] X.Q. Liu, S. Jiang, W.B. Fan, W.M. Liu, Commun. Nonlin. Sci. Numer. Simulat. 9, 361 (2004).

[21] H.H. Chen, C.S. Liu, Phys. Rev. Lett. 37, 693 (1976).

[22] H.M. Li, F.M. Wu, Chin. Phys. Lett. 21, 1425 (2004). 